\title{
Metazoan Endoparasites of Three Species of Anurans Collected from the Middle Black Sea Region of Turkey
}

\author{
S. DÜŞEN ${ }^{1,3}$, M. C. OĞUZ ${ }^{2}$ \\ ${ }^{1}$ Pamukkale University, Faculty of Arts and Sciences, Department of Biology, Kinikli Campus, Kinikli, \\ 20017 Denizli, Turkey, E-mail: sdusen@pamukkale.edu.tr, serdar2290@yahoo.com; ${ }^{2}$ Atatürk University, \\ Faculty of Arts and Sciences, Department of Biology, Campus, 25240 Erzurum, Turkey
}

\begin{abstract}
Summary
In this report, European common toads; Bufo bufo, European green toads Bufo viridis and marsh frogs Rana ridibunda were collected in Amasya, Çorum, and Tokat Provinces (Middle Black Sea Region of Turkey) 2005 and 2006 and examined for helminths. Two of $2(100 \%)$ Bufo bufo and 8 of 8 (100\%) Bufo viridis and 57 of 63 $(90.5 \%)$ Rana ridibunda were infected with 1 or more helminths. The helminths of B. bufo included Oswaldocruzia filiformis and Oxysomatium brevicaudatum. The helminth fauna of $B$. viridis comprised 5 species: 1 species of trematode (Pleurogenoides medians), 1 species of cestode (Nematotaenia dispar) and 3 species of nematodes, (Oswaldocruzia filiformis, Cosmocerca ornata, and Oxysomatium brevicaudatum), while the helminth fauna of Rana ridibunda comprised 9 species: 4 species of trematodes (Gorgodera cygnoides, Gorgoderina vitelliloba, Haematoloechus breviansa, and Opisthioglyphe ranae), 3 species of nematodes Oswaldocruzia filiformis, Cosmocerca ornata, and Oxysomatium brevicaudatum), and 2 species of acanthocephalans (Pomphorhynchus laevis and Acanthocephalus ranae). Oswaldocruzia filiformis, Cosmocerca ornata, and Oxysomatium brevicaudatum were collected from all three host species. In addition, Pleurogenoides medians represents a new host record for Bufo viridis in Turkey.
\end{abstract}

Keywords: Bufo bufo; Bufo viridis; Rana ridibunda; endoparasites; helminths; Middle Black Sea Region; Turkey

\section{Introduction}

Little information is available on the helminths of anurans in the northern parts of Turkey. Yildirımhan et al. (2005a) reported 3 species of Nematoda and 1 species of Acanthocephala in the marsh frog, Rana ridibunda collected from the Rize Province. Also, Yildırımhan and Karadeniz
(2007) reported 5 species of Nematoda, and 1 species of Acanthocephala in European common toad, B. bufo, from Trabzon Province, (northeast part of Turkey). To our knowledge, there are no reports of helminths of Bufo bufo, European green toads, Bufo viridis, and $R$. ridibunda from Amasya, Çorum and Tokat provinces (Middle Black Sea Region of Turkey). Therefore, this is the first report of helminths collected from 3 species of the anuran community of these Turkish provinces.

\section{Material and methods}

Seventy-three anurans representing 3 species were collected between 2005 - 2006. Two B. bufo (males), mean snout-vent length $(\mathrm{SVL})=80.5 \mathrm{~mm}, 8$ B. viridis $(6$ males and 2 females), $80 \pm 1.8 \mathrm{~mm}$ and $63 R$. ridibunda (37 males and 26 females), $7.5 \pm 1.8 \mathrm{~mm}$ were collected by dip net and hand from the Yeșilırmak River (Amasya Province, $\left.40^{\circ} 40^{\prime} \mathrm{N} ; 35^{\circ} 50^{\prime} \mathrm{E}\right)$, Tokat Province (40 ${ }^{\circ} 18^{\prime}$ $\left.\mathrm{N} ; 36^{\circ} 33^{\prime} \mathrm{E}\right)$ and Çorum Province $\left(40^{\circ} 33^{\prime} \mathrm{N} ; 34^{\circ} 57^{\prime} \mathrm{E}\right)$. Within $48 \mathrm{hr}$, frogs were overdosed in ether-filled glass containers. The body cavity was opened by a longitidutinal ventral incision. The alimentary canal was excised and separated into stomach, small intestine, large intestine and rectum. The contents of each part and other organs (lungs, liver, gall bladder, kidneys and urinary bladder) were each mixed with $0.5 \%$ saline solution and poured into petri dishes for examination under a stereomicroscope. The muscles, plus portions of peritoneum and spinal cord, were teased out with needles and examined under a stereomicroscope. Trematodes were immobilized by heat, fixed, and stored in $70 \%$ ethanol. Nematodes were straightened by heat, fixed, and stored in $70 \%$ ethanol with $5 \%$ glycerol. Acanthocephalans were relaxed in saline and heat-fixed under slight coverslip pressure in warm alcohol-formalinacetic acid. Digeneans and acanthocephalans were stained 
with acetocarmine, dehydrated, cleared in cedar oil or xylol, and mounted in Canada Balsam; nematodes were cleared in glycerol and examined. Intensities are presented as mean values ( $\pm 1 \mathrm{SD}$ ) followed by the range.

Voucher specimens of parasites were deposited in the Ege University Museum of Zoology, Izmir, Turkey (ZDEU HEL-5-15/2007); host voucher specimens were deposited in the Atatürk University Department of Biology, Erzurum, Turkey.

\section{Results and Discussion}

A total of 73 anurans from 3 species Bufo bufo, Bufo viridis and Rana ridibunda were investigated. In summary, 541 individuals of 11 helminth species were collected from $66(91.8 \%)$ of the 73 anurans examined. Helminths were observed from the intestine, urinary bladder and lungs of these species (Table 1).

No individual host harbored more than 4 helminth species. Of the infected anurans, 24 (36.4\%), harbored 1 species of helminth; 25 (37.9\%), harbored 2 species of helminth; $24(10.6 \%)$, harbored 3 species of helminth and $1(1.5 \%)$ harbored 4 species of helminth. There were $1.74 \pm 0.74$ helminth species per infected host and $9.13 \pm 7.50$ helminth individuals per infected host. Of the 11 helminth species found in this study, only 4 helminths had prevalences greater than $10 \%, C$. ornata was the most prevalent helminth, occurring in 40 of 73 hosts $(54.8 \%)$, followed by $O$. ranae with 24 of 73 hosts $(32.9 \%)$, O. brevicaudatum with 19 of 73 hosts $(26.0 \%)$, and $A$. ranae with 9 of 73 hosts $(12.3 \%)$.

\section{Family Gorgoderidae}

Gorgodera cygnoides (Zeder, 1800) Looss, 1899

(Syns. Distoma cygnoides Zeder, 1800; Distomum cygnoides Looss, 1894; Gorgodera loosi Sinitzin, 1905) G. cygnoides is a common parasite of amphibians, Bombina bombina (Vojtková \& Vojtek, 1975); B. variegata, (Vojtková \& Vojtek, 1975; Buchvarov, 1977); Bombinator igneus (Yamaguti, 1958); Bufo viridis (Buchvarov, 1977; Shimalov \& Shimalov, 2001); Hyla arborea (Yamaguti, 1958); Rana arvalis (Vojtková \& Vojtek, 1975); R. dalmatina (Buchvarov, 1983), R. esculenta (Yamaguti, 1958; Vojtková \& Vojtek, 1975; Buchvarov, 1977; Kuc \& Sulgostowska, 1988b); R. lessonae (Vojtková \& Vojtek, 1975); R. clamitans (Yamaguti, 1958); R. temporaria (Yamaguti, 1958; Vojtková \& Vojtek, 1975); R. ridibunda (Kuc \& Sulgostowska, 1988a; Kirin 2002b); R. camerani (Yıldırımhan et al., 2006a) and R. kurtmuelleri (Hristovski et al., 2006). G. cygnoides was reported several papers in Urinary bladder of $R$. ridibunda from Turkey (Yıldırımhan et al., 1996; Yıldırımhan et al., 2005; Düşen \& Öz, 2006). The geographic range of this species is Western Europe (Yamaguti, 1958).

Specimens deposited: (ZDEU HEL-5/2007)

Gorgoderina vitelliloba (Olsson, 1876) Looss, 1902 (Syn. Distoma vitellilobum Olsson, 1876)
G. vitelliloba was reported, Bombinator igneus (Yamaguti, 1958); B. bombina (Vojtková \& Vojtek, 1975); B. variegata, (Yamaguti, 1958; Vojtková \& Vojtek, 1975; Buchvarov, 1977) B. bufo and B. calamita (Cedhagen, 1988); B. viridis (Buchvarov, 1977); R. arvalis (Yamaguti, 1958; Vojtková \& Vojtek, 1975); R. dalmatina (Buchvarov, 1977); R. esculenta, (Vojtková \& Vojtek, 1975); R. macrocnemis (Yıldırımhan et al., 1997b; Yildırımhan et al., 2006a); and R. temporaria (Yamaguti, 1958; Vojtková \& Vojtek, 1975; Buchvarov, 1977; Cedhagen, 1988).

Similiarly G. cygnoides, G. vitelliloba has been recorded by different researchers in $R$. ridibunda from Turkey (Y11dırımhan et al., 1996; Yıldırımhan et al., 2005; Düşen \& Öz, 2006; Sağlam \& Arıkan, 2006). Geographic range of this species, Europe and Asia minor (Yamaguti, 1958), Czech Republic (Vojtková \& Vojtek, 1975), Bulgaria (Batchvarov et al., 1975; Buchvarov, 1977), Turkey (Yildirımhan et al., 1996).

Specimens deposited: (ZDEU HEL-6/2007)

Family Lecithodendriidae

Pleurogenoides medians (Olsson, 1876) Travassos, 1921

(Syns. Distomum medians Olsson, 1876; Pleurogenes medians (Olsson, 1876))

$P$. medians has been reported in various amphibians and reptiles species, Triturus cristatus (Shimalov et al., 2001); T. vulgaris (Vojtková \& Vojtek, 1975; Shimalov et al., 2001); B. bombina, (Vojtková \& Vojtek, 1975); B. variegata, (Vojtková \& Vojtek, 1975; Shimalov \& Shimalov, 2001); B. bufo (Shimalov \& Shimalov, 2000); B. calamita, (Vojtková \& Vojtek, 1975); B. vulgaris (Yamaguti, 1958); H. arborea (Vojtková \& Vojtek, 1975; Düşen \& Öz, 2004); R. arvalis (Vojtková \& Vojtek, 1975); R. camerani (Yıldırımhan et al., 2006a; Düşen, 2007); R. dalmatina (Buchvarov, 1977); R. esculenta (Vojtková \& Vojtek, 1975; Buchvarov, 1977, Kuc \& Sulgostowska, 1988b); $R$. macrocnemis (Y1ldırımhan et al., 2006b, Düşen, 2007); $R$. ridibunda (Buchvarov, 1977; Kuc \& Sulgostowska, 1988a; Oğuz et al., 1994; Yıldırımhan et al., 1996; Mashaii et al., 2000; Yıldırımhan et al., 2005a; Düşen \& Öz, 2006; Sağlam \& Arıkan, 2006); R. arvalis (Vojtková \& Vojtek, 1975); R. temporaria (Vojtková \& Vojtek, 1975; Cedhagen, 1977); and Lacerta trilineata (Yamaguti, 1963; Y1ld1rimhan, 1999b).

The geographic range of $P$. medians is Europe and Asia (Yamaguti, 1958); Austrolasian Regions (Prudhoe \& Bray, 1982). P. medians represent a new parasite record for Bufo viridis in Turkey, similiarly, Buchvarov (1977) reported this helminth the small intestine of $B$. viridis from Bulgaria.

Specimens deposited: (ZDEU HEL-7/2007)

Family Plagiorchiidae

Haematoloechus breviansa (Sudarikov, 1950)

Sey and Eory (1992) reported H. breviansa in R. ridibunda from Hungary, Mashaii et al. (2000) reported $H$. breviansa in $R$. ridibunda from Northern parts of Iran. Y1ldırımhan et al. (2005) and Düşen and Öz, (2006) recorded this 
Table 1. Helminths of three frogs species of Middle Black Sea Region, Turkey

\begin{tabular}{|c|c|c|c|c|}
\hline Host species & $\mathbf{N}$ & In \& (\%) & Identified helminths group & $\operatorname{Max}(\mathrm{Me}) \sum$ \\
\hline Bufo bufo & 2 & $2(100 \%)$ & Oswaldocruzia filiformis & $1(1) 1$ \\
\hline Bufo bufo & 2 & $2(100 \%)$ & Oxysomatium brevicaudatum & $1(1) 1$ \\
\hline Bufo viridis & 8 & $1(12.5 \%)$ & Pleurogenoides medians & $1(1) 1$ \\
\hline Bufo viridis & 8 & $5(62.5 \%)$ & Nematotaenia dispar & $12(5.4) 27$ \\
\hline Bufo viridis & 8 & $1(12.5 \%)$ & Oswaldocruzia filiformis & $3(1) 3$ \\
\hline Bufo viridis & 8 & $4(50 \%)$ & Oxysomatium brevicaudatum & $22(9.3) 37$ \\
\hline Bufo viridis & 8 & $2(25 \%)$ & Cosmocerca ornata & $2(1.5) 3$ \\
\hline Rana ridibunda & 63 & $5(8 \%)$ & Gorgodera cygnoides & $4(2) 10$ \\
\hline Rana ridibunda & 63 & $1(1.6 \%)$ & Gorgoderina vitelliloba & $5(1) 5$ \\
\hline Rana ridibunda & 63 & $2(3.2 \%)$ & Haematoloechus breviansa & $2(2) 4$ \\
\hline Rana ridibunda & 63 & $24(38 \%)$ & Opisthioglyphe ranae & $22(5) 121$ \\
\hline Rana ridibunda & 63 & $3(4.8 \%)$ & Oswaldocruzia filiformis & $3(2) 6$ \\
\hline Rana ridibunda & 63 & $14(22.2 \%)$ & Oxysomatium brevicaudatum & $9(4.4) 61$ \\
\hline Rana ridibunda & 63 & $38(60.3 \%)$ & Cosmocerca ornata & $30(7.3) 277$ \\
\hline Rana ridibunda & 63 & $1(1.6 \%)$ & Pomphorhynchus laevis & $1(1) 1$ \\
\hline Rana ridibunda & 63 & $9(14.3 \%)$ & Acanthocephalus ranae & $9(3.4) 31$ \\
\hline
\end{tabular}

helminths in the lungs of $R$. ridibunda from Turkey. The geographic range of $H$. breviansa, Russia (Yamaguti, 1958), Turkey (Yıldırımhan et al., 1996), Iran (Mashaii et al., 2000).

Specimens deposited: (ZDEU HEL-8/2007)

Opisthioglyphe ranae (Froelich, 1791)

(Syn. Opisthioglyphe endoloba (Dujardin, 1845) Looss, 1899)

The reported hosts of $O$. ranae were, Salamandra maculosa (Vojtková \& Vojtek, 1975; Buchvarov, 1977); T. cristatus (Yamaguti, 1958; Shimalov et al., 2001); T. vulgaris (Shimalov et al., 2001); B. bombina and B. variegata (Vojtková \& Vojtek, 1975; Yıldırımhan et al., 2001a); $R$. ridibunda (Batchvarov et al., 1975; Kuc \& Sulgostowska, 1988a; Fernando, 1989; Masshai et al., 2000; Kirin, 2003a, b); B. bufo (Shimalov \& Shimalov, 2001); B. calamita (Yamaguti, 1958; Shimalov \& Shimalov, 2001); B. vulgaris, B. variabilis, (Yamaguti, 1958); B. viridis, (Vojtková \& Vojtek, 1975; Buchvarov, 1977); H. arborea (Vojtková \& Vojtek, 1975); Pelobates fuscus (Vojtková \& Vojtek, 1975); R. dalmatina (Vojtková \& Vojtek, 1975; Buchvarov, 1977); $R$. esculenta (Yamaguti, 1958; Vojtková \& Vojtek, 1975; Buchvarov, 1977); R. lessonae (Vojtková \& Vojtek, 1975); R. temporaria (Yamaguti, 1958; Vojtková \& Vojtek, 1975; Buchvarov, 1977); R. arvalis (Vojtková \& Vojtek, 1975); Natrix natrix (Shimalov \& Shimalov, 2000) and $V$. berus (Shimalov \& Shimalov, 2000).

Geographic range of $O$. ranae, Europe (Yamaguti, 1958), Turkey (Yıldırımhan et al., 1996), Saudi Arabia (Fernando,
1989), Iran (Masshai et al., 2000).

Specimens deposited: (ZDEU HEL-9/2007)

Family Nematotaeniidae

Nematotaenia dispar (Goeze, 1782) Lühe, 1899

(Syns. Taenia dispar Goeze, 1782; Taenia dispar salamandrae Frölich, 1789; Taenia bufonis Gmelin, 1790; Halysis obvoluta Zeder, 1803; Nematotaenia kashmirensis Fotedar, 1966; Nematotaenia dollfusi Yuen and Fernando, 1974; Nematotaenia viride Mokhtar-Maamouri and Chakroun, 1984)

$N$. dispar is commonly parasitic in the intestine of amphibians, rarely reptiles, of the Oriental, Nearctic, Palearctic (Prudhoe \& Bray, 1982); Mertensiella caucasica (Y1ld1rimhan et al., 2005b); B. bombina (Buchvarov, 1977); B. variegata (Buchvarov, 1977; Prudhoe \& Bray, 1982); $B$. bufo (Prudhoe \& Bray, 1982); B. viridis (Mashaii, 2005); B. alvarius (Goldberg \& Bursey, 1991); H. arborea (Buchvarov, 1977, Prudhoe \& Bray, 1982; Vashetko \& Siddikkov, 1999); H. savignyi (Al-Sorkhy \& Amr, 2003; Mashaii, 2005); and $R$. ridibunda, $R$. temporaria (Buchvarov, 1977); R. bedriagae (Al-Sorky \& Amr, 2003). Yıldırımhan (1999) reported $N$. dispar in B. viridis from Turkey.

Specimens deposited: (ZDEU HEL-10/2007)

Family Molineidae

Oswaldocruzia filiformis (Goeze, 1782) Travassos, 1917

(Syns. Ascaris filiformis, Goeze, 1782; Cucullanus ranae Goeze, 1782; Ascaris tennissima Schark, 1788; Ascaris intestinalis Gmelin, 1790; Ascaris bufonis Gmelin, 1790; 
Strongylus auricularis Zeder, 1800; Ascaris setiformis Goeze in Zeder, 1800; Strongylus dispar Dujardin, 1845; Oswaldocruzia insulae Morishita, 1923; Strongyluris bialata Molin, 1861; Oswaldocruzia molgeta Lewis, 1928; Oswaldocruzia skrjabini Travassos, 1937; Oswaldocruzia problematica Ivanitzky, 1940; Oswaldocruzia goezi Skrjabin and Schulz, 1952)

$O$. filiformis appear to be that known various amphibian and reptiles species, S. salamandra (Buchvarov, 1977); T. alpestris, T. karelini (Buchvarov, 1977; Cedhagen, 1988; Kirin \& Buchvarov, 2002); T. vulgaris (Buchvarov, 1977; Shimalov et al., 2001); Bombina bombina, B. variegata (Buchvarov, 1977; Kirin \& Buchvarov, 2002); B. regularis (Schad et al., 1960); B. viridis (Schad et al., 1960; Batchvarov et al., 1975; Buchvarov, 1977; Vashetko \& Sidd1kov, 1999; Yildırımhan, 1999a; Shimalov \& Shimalov, 2001); H. arborea (Buchvarov, 1977; Y1ldırımhan et al., 2006c); R. arvalis (Yıldırımhan et al., 2006a); R. camerani (Yıldırımhan et al., 2006a); R. dalmatina, (Batchvarov et al., 1975; Buchvarov, 1977; Kirin \& Buchvarov, 2002); $R$. kurtmuelleri (Hristovski et al., 2006); R. macrocnemis (Schad et al., 1960; Yıldırımhan et al., 1997a; Yıldırımhan et al., 2006b); R. ridibunda (Buchvarov, 1977; Y1ldır1mhan et al., 1996; Batchvarov et al., 1975; Kirin \& Buchvarov, 2002; Y1ldırımhan et al., 2005a; Sağlam and Ar1kan, 2006); Rana temporaria, (Buchvarov, 1977; Cedhagen, 1988; Kirin \& Buchvarov, 2002); R. graeca (Božkov \& Stojkova, 1970; Buchvarov, 1977); Lacerta agilis, (Shimalov et al., 2000; Mihalca et al., 2007); L. trilineata (Yıldırımhan, 1999b); L. viridis (Biserkov \& Kostadinova, 1998; Yıldırımhan 1999b; Kirin, 2002a; Borkovcová \& Kopřiva, 2005); L. vivipara (Shimalov et al., 2000); Anguis fragilis (Schad et al., 1960; Bertman \& Okulewicz, 1987; Shimalov et al., 2000; Borkovcová \& Kopřiva, 2005); Zootoca vivipara (Sanchis et al., 2000); N. natrix (Bertman \& Okulewicz, 1987; Shimalov \& Shimalov, 2000) and V. berus (Shimalov \& Shimalov, 2000).

Schad et al (1960) were first time reported O. filiformis in Bufo regularis and $R$. macrocnemis from Turkey. We observed this species in small and large intestines from three frogs species in this study. The geographic range of O. filiformis is Europe and Asia (Yamaguti, 1961). Specimens deposited: (ZDEU HEL-11/2007)

\section{Family Cosmocercidae}

Oxysomatium brevicaudatum (Zeder, 1800) Railliet and Henry, 1916

(Syns. Neoxysomatium brevicaudatum (Zeder, 1800), Fusaria brevicaudata Zeder, 1800; Oxysomatium longispiculum Railliet and Henry, 1916, Oxysoma contortum Linstow, 1906)

O. brevicaudatum was reported different hosts of amphibians and reptiles in Europe and Asia; Bombina, Bufo, Hyla, Pelobates, Rana, Salamandra (Yamaguti, 1961); S. salamandra, S. atra, Pseudotriton ruber and B. bombina (Walton, 1933); (Walton, 1933); B. bufo (Walton, 1933; Shimalov \& Shimalov, 2001; Galli et al, 2001); B. viridis (Walton, 1933; Schad et al., 1960; Buchvarov, 1977;
Y1ldırımhan, 1999a); B. regularis (Schad et al., 1960); $H$. arborea (Walton, 1933); R. dalmatina (Buchvarov, 1977); R. graeca (Božkov \& Stojkova, 1970; Buchvarov, 1977); R. esculenta (Walton, 1933); R. kurtmuelleri (Hristovski et al., 2006); R. macrocnemis (Schad et al., 1960); $R$. ridibunda (Schad et al., 1960; Kirin \& Buchvarov, 2002; Y11dırımhan et al., 2005a; Sağlam \& Arıkan, 2006); R. temporaria (Walton, 1933; Buchvarov, 1977; Kirin \& Buchvarov, 2002); P. fuscus (Walton, 1933); A. fragilis (Schad et al., 1960; Shimalov et al., 2000; Sharpilo, 2003; Borkovcová \& Kopřiva, 2005); N. natrix (Schad et al., 1960; Shimalov \& Shimalov, 2000) and V. berus (Shimalov \& Shimalov, 2000).

Schad et al (1960) were first time reported this helminth in Turkey. Sharpilo (2003) pointed out that O. brevicaudatum has a great disperse in $A$. fragilis in Caucasican Region. $O$. brevicaudatum was observed from three frogs species intestines in this research.

Specimens deposited: (ZDEU HEL-12/2007)

Cosmocerca ornata (Dujardin, 1845)

(Syns. Oxyuris ornata (Dujardin, 1845) Schneider, 1866; Anancous commutatus Railliet and Henry, 1976; Cosmocerca minuscule Travassos, 1931; Paracosmocerca mucronata Kung and $\mathrm{Wu}, 1945$; Cosmocercella polissensis Maguzo, 1972; Cosmocerca indica Nama and Khichi, 1973; Paracosmocerca spinocerca Rao, 1979; Cosmocerca macrogubernaculum Rao, 1979)

There are several papers reporting $C$. ornata from many species of amphibians and reptiles, Bufo, Hyla, Rana, Triturus (Yamaguti, 1961); T. alpestris (Walton, 1933; Buchvarov, 1977; Shimalov et al., 2000); T. cristatus (Walton, 1933; Shimalov et al., 2001, T. vulgaris (Shimalov et al., 2001); B. bombina, (Buchvarov, 1977; Grabda-Kazubska \& Lewin, 1989); B. variegata (Buchvarov, 1977; Grabda-Kazubska \& Lewin, 1989; Kirin \& Buchvarov, 2002); B. bufo (Walton, 1933; Buchvarov, 1977; Shimalov \& Shimalov 2001; Galli et al., 2000; Galli et al., 2001); B. viridis (Batchvarov et al., 1975; Buchvarov, 1977; Vashetko \& Siddikov, 1999; Mashaii, 2005); $H$. arborea (Buchvarov, 1977; Y1ldırımhan et al., 2006c); $P$. syriacus (Shimalov et al., 2000); R. esculenta (Walton, 1933; Buchvarov, 1977); R. arvalis (Cedhagen, 1988; Kuc \& Sulgostowska, 1988b); R. temporaria, (Walton, 1933; Buchvarov, 1977; Kuc \& Sulgostowska, 1988b); R. graeca (Božkov \& Stojkova, 1970; Buchvarov, 1977); R. holtzi (Yildırımhan et al., 2006b); R. macrocnemis (Yildırımhan et al., 2006b; Düşen, 2007); R. ridibunda (Batchvarov et al., 1975; Buchvarov, 1977; Kuc \& Sulgostowska, 1988a; Mashaii et al., 2000; Kirin \& Buchvarov, 2002; Kirin, 2003a, b; Yıldırımhan et al., 2005a; Düşen, 2006); $R$. camerani (Yıldırımhan et al 2006a; Düşen 2007); Chiasmocleis capixaba (Van Sluys et al., 2006) and A. fragilis (Shimalov et al., 2000). Schad et al (1960) were first time reported $C$. ornata in B. viridis, $R$. macrocnemis and $R$. ridibunda from Turkey. The Geographic range of $C$. ornata New and Old Worlds (Baker, 1987).

Specimens deposited: (ZDEU HEL-13/2007) 
Family Pomphorhynchidae

Pomphorhynchus laevis (Zoega in Müller 1776)

(Syns. Echinorhynchus salvelini Schrank, 1788; Echinorhynchus nodulosus Schrank, 1790; Echinorhynchus annulatus Gmelin, 1791; Echinorhynchus barbi Schrank, 1792; Echinorhynchus gobii Viborg, 1795; Echinorhynchus proteus Westrumb, 1821; Acanthocephalus lavareti Koelreuter, 1771)

$P$. laevis, occurs predominantly in cyprinid and also in salmonid fishes with the Palaearctic distribution (Dudiňák \& Šnábel, 2001). P. laevis, is recorded in several fish species in Turkey: barbel (Barbus plebejus escherichii), bleak (Alburnus alburnus), chub (Leuciscus cephalus), common carp (Cyprinus carpio), crucian carp (Carassius carassius), loach (Nemachilus sp.), nase (Chondrostoma nasus), pike (Esox lucius), sheatfish (Silurus glanis), (Öktener, 2003), and tench (Tinca tinca) (Yıldız, 2003; Yıldız \& Çavuşoğlu, 2003).

A number of marine and freshwater fishes have been reported as definitive hosts of acanthocephalan $P$. laevis (Ziolkowska \& Rokicki, 2003). Buchvarov (1977) observed in Rana ridibunda and Veith and Erpelding (1995) in fire salamander (Salamandra salamandra) are reported $P$. laevis. So far, there have been no published studies on $P$. laevis, for frog species, which are distributed in Turkey. Specimens deposited: (ZDEU HEL-14/2007)

\section{Family Echinorhynchidae}

Acanthocephalus ranae (Schrank, 1788) Lühe, 1911

(Syns. Echinorhynchus ranae Schrank, 1788; Echinorhynchus haeruca Rudolphi, 1808)

A number of amphibians and reptiles species have been reported hosts of acanthocephalan A. ranae; Bombinator sp., Diemictylus viridescens, Hyla sp., Rana sp., Triturus sp., Salamandra sp. (Yamaguti, 1963); M. caucasica (Y1ld1rimhan et al., 2005b); B. calamita (Shimalov \& Shimalov, 2001); B. viridis (Buchvarov, 1977; Y1ldırımhan, 1999a; Vashetko \& Siddikov 1999; Shimalov \& Shimalov, 2001); B. bombina (Buchvarov, 1977; Grabda-Kazubska \& Lewin, 1989; Y1ldırımhan et al., 2001a); B. variegata (Grabda-Kazubska \& Lewin, 1989); H. arborea (Düşen \& Öz, 2004); R. arvalis (Buchvarov, 1977; Cedhagen, 1988; Kuc \& Sulgostowska, 1988b); R. dalmatina (Buchvarov, 1977); $R$. esculenta (Buchvarov, 1977; Kuc \& Sulgostowska, 1988b); R. temporaria (Buchvarov, 1977; Cedhagen, 1988; Kuc \& Sulgostowska, 1988b); R. kurtmuelleri (Hristovski et al., 2006); R. ridibunda (Buchvarov, 1977; Kuc \& Sulgostowska, 1988b; Sey \& Eory, 1992); R. camerani (Yıld1rımhan et al., 2006a); R. macrocnemis (Y1ldırımhan et al., 1997a; Yıldırımhan et al., 2006b; Düşen, 2007); A. fragilis (Shimalov et al., 2000); N. natrix (Yamaguti, 1963; Shimalov \& Shimalov, 2000).

Oğuz et al. (1994), Yıldırımhan et al. (1996), Yildırımhan et al. (2005a), Düşen and Öz, (2006), and Sağlam and Ar1kan (2006) recorded $A$. ranae in $R$. ridibunda from Turkey. The geographic range of this helminth, Europe, U. S. A., Russia (Yamaguti, 1963); Turkey (Oğuz et al., 1994). Specimens deposited: (ZDEU HEL-15/2007)
This is the first published study of helminths of Bufo bufo, Bufo viridis, and Rana ridibunda from Middle Black Sea Region of Turkey. Yildirımhan et al. (2005a) reported 3 species of Nematoda (O. filiformis, $C$. ornata and $O$. brevicaudatum) and 1 species of Acanthocephala (Acanthocephalus ranae) in $R$. ridibunda collected from the Rize Province. Also, Yıldırımhan and Karadeniz (2007) reported in $B$. bufo, from Trabzon Province, 5 species of Nematoda: Aplectana acuminata, A. macintoshii, C. ornata, O. filiformis, and Rhabdias bufonis, and 1 species of Acanthocephala, Acanthocephalus ranae.

In both investigations (Y1ldirimhan et al., 2005a and Y1ldırımhan \& Karadeniz, 2007), C. ornata was the most prevalent parasite of hosts. Similiarly, in this study $C$. ornata was observed the most prevalent parasite. Gorgodera cygnoides, Gorgoderina vitelliloba, Haematoloechus breviansa, Opisthioglyphe ranae and Pomphorhynchus laevis were not recorded by Yildirımhan et al. (2005a) and helminths reported by Yildirimhan et al. (2005a), not observed in this study were Aplectana acuminata, A. macintoshii, Rhabdias bufonis.

The trematode Pleurogenoides medians represents a new host record for $B$. viridis in Turkey. Further helminthological investigations are needed in amphibians from Turkey, to add other metazoan endoparasites to Turkish amphibian helminthofauna.

\section{Acknowledgments}

This study supported by the Atatürk University Scientific Research Projects Unit (Project No: BAP-2005/74). We are indebted to Atatürk University Scientific Research Projects Unit for financial support. We should also like to thank, for their permission and help, the Department of National Parks and Wildlife of the Ministry of Forestry, of the Republic of Turkey.

\section{References}

AL-SorkHY, M. K., AMr, Z. (2003): Platyhelminth parasites of some amhibians in Jordan. Turk. J. Zool., 27: 89 - 93 Bertman, M., OKUlEwiCZ, A. (1987): Lizards (Anguis fragilis L.) and snakes (Natrix natrix (L.)) as new hosts of Oswaldocruzia filiformis (Goeze, 1782) Travassos 1917 (Nematoda). Wiad. Parazytol., 33 (2): 209 - 212 (In Polish)

Biserkov, V., Kostadinova, A. (1998): Intestinal helminth communities in the green lizard, Lacerta viridis, from Bulgaria. J. Helminthol., 72 (3): 267 - 271. DOI: 10.1017/S0022149X00016540

BorkovCOVÁ, M., KOPŘIVA, J. (2005): Parasitic helminths of reptiles (Reptilia) South Moravia (Czech Republic). Parasitol. Res., 95: 77 - 78. DOI: 10.1007/s00436-004-12 58-6

BožKov, D., StojKovA, R. (1970): Beitrag zur untersuchung der Helminthenfauna der Rana graeca in Bulgarien. Bulletin de L'institute de Zoologie et Musée, Academie Bulgare des Sciences, pp. 69 - 75 (In Bulgarian) 
Buchvarov, G. K. (1977): Catalogue des Helminthes des Amphibies en Bulgarie, Universite de Plovdiv „P Hilendarski,“ Plovdiv, Bulgaria. 53 pp. (In Bulgarian)

Buchyarov, G. K., Petrov, P., Chochev, B. (1975): To the question about helminthofauna of Amphibious Eucaudate (Amphibia-Eucaudata) of Velingrad's District. Universite de Plovdiv "P Hilendarski", Travaux Scientifiques, Biologie, 13(4): 53 - 64

BuchVarov, G. K. 1983. Apport l'etude de helminthofaune des Amphipies sons queue (Amphibia-ecadata) Du bassin de fleuve de la strouma. Nachne Trudove, Universite' de Plovdiv "Paissi Hilendarski'" Travaux Scientifiques, 21: $373-380$

Cedhagen, T. (1988): Endoparasites in some Swedish amphibians. Acta Parasitol., 33: 107 - 113

DUDIŇÁK, V., ŠNÁBEL, V. (2001): Comparative Analysis of Slovak and Czech Populations of Pomphorhynchus laevis (Acanthocephala) Using Morphological and Isoenzyme Analyses. Acta Zool. Univ. Comenianae, 44: 41 - 50 DÜŞEN S., ÖZ, M. (2004): Helminth parasites of the tree frog, Hyla arborea (Linnaeus, 1758) (Anura: Hylidae) from Southwest Turkey. Comp. Parasitol., 71 (2): 258261. DOI: $10.1654 / 4123$

DÜŞEN S., Öz, M. (2006): Parasitic helminths of the marsh frog, Rana ridibunda Pallas, 1771 (Anura: Ranidae), from Antalya Province, south-west Turkey. Comp. Parasitol., 73 (1): 121 - 129. DOI: 10.1654/4162.1

DÜŞEN, S. (2007): Helminths of the two mountain frogs, Banded frog, Rana camerani Boulenger, 1886 and Uludağ Frog Rana macrocnemis Boulenger, 1885 (Anura: Ranidae), collected from Antalya Province. Acta Parasitol. Tur., 31 (1): $84-88$

FERnANDO, M. M. (1989): The parasitic burden of the frog Rana ridibunda Pallas, 1771 from Saudia Arabia. A preliminary list of parasitic helminths. Herpetol. J., 1: $415-$ 417

Galli, P., Gentilli, A., Santagostino, M., Crosa, G. (2000): Parasites community from Bufo bufo killed by traffic. Third Conference Safeguard The Amphibians. June, 23 - 24, Lugano, Cantone Ticino - Switzerland.

Galli, P., Crosa, G., Gentilli, A., Santagostino, M. (2001): New geographical records of parasitic nematodes from Bufo bufo in Italy. Parassitologia., 43 (4): 147 - 149 GRABDA-KAZUBSKA B., LEWIN, J. (1989): The helminth fauna of Bombina bombina (L.) and Bombina variegata (L.) in Poland. Acta Parasitol., 34: 273 - 279

Hristovski, N., Smilkov, S., TomovskA, D., Popovich E., Kostich, D. (2006): Helmintofauna of Rana kurtmuelleri Gayda, 1940 syn. Rana balcanica Shneider et Sinish, 1992 (Anura:Ranidae) from Dojran Lake, Makedonia and Greece. $10^{\text {th }}$ International Congress on the Zoogeography and Ecology of Greece and Adjacent Regions Patras, Greece. June 26 - 30

KIRIN, D. (2002a): New data on the helminth fauna of Lacerta viridis Laurenti, 1768, and Podarcis muralis (Laurenti, 1768) (Reptilia: Lacertidae) in Bulgaria. Acta Zool. Bulg., 54 (1): 43 - 48

KIRIN, D. (2002b): Biodiversity and Trematod assemblages in Rana ridibunda Pallas from the district of Troyan Town. Exp. Pathol. Parasitol., 5 (8): 7 - 12

KIRIN, D., BuchVarov, G. (2002): Biodiversity of of the helminth communities of acaudated Amphibians (Amphibia: Ecaudata) from Bistritsa Riverside (Gotse Delchev Region). Exp. Pathol. Parasitol., 5 (8): 13 - 16

KIRIN, D. (2003a): Biodiversity and ecological particulars of the helminth communities in Rana ridibunda Pallas, 1771, from districts of town Saedinenie. Exp. Pathol. Parasitol., 6 (11): 31-36

KIRIN, D. (2003b): Biological diversity and ecological measurements of the helminth communities of Rana ridibunda from District of Town Vidin. Exp. Pathol. Parasitol., 6 (11): $37-43$

KuC, I., SulgostowskA, T. (1988a): Helminth fauna of Rana ridibunda Pallas, 1771 from Goclawski Canal in Warszaw (Poland). Acta Parasitol., 33: 101 - 105

KuC, I., SulgostowskA, T. (1988b): Helminth fauna of frogs in the forest of Kampinos near Warszawa. Acta Parasitol., 33: $267-272$

Mashait, N., BAlOuCH, M., Mobedi, I. (2000): New records about helminth parasites of the marsh frog, Rana ridibunda (Anura: Ranidae), from the North of Iran. Iran. J. Fish. Sci., 2: $77-88$

MASHAII, N. (2005): Helminth Parasites of green toad, Bufo viridis (Anura: Bufonidae), Tree frog, Hyla arborea savignyi (Anura: Hylidae) and Marsh Frog, Rana ridibunda ridibunda (Anura: Ranidae) from Southwest of Iran. Iran. J. Vet. Res., 6 (3): $67-73$

Mihalca, A. D., Gherman, C., Ghira , I., Cozma, V. (2007): Helminth parasites of reptiles (Reptilia) in Romania. Parasitol. Res., 95: $77-78$

OĞUz, M. C., Altunel, F. N., UĞURTaş, İ. H. (1994): An investigation of the species of Plathelminthes and Acanthocephalus ranae (Schrank, 1788, Acanthocephala) of marsh frogs (Rana ridibunda Pallas, 1771) which were collected from the Bursa and Edirne Regions. Tr. J. of Zool., 18: 47 - 51 (In Turkish, with English abstract)

ÖKTENER, A. (2003): A checklist of Metazoan Parasites Recorded in Freshwater Fish from Turkey. Zootaxa, 394: $1-28$

Prudhoe, S., A. Bray, S. (1982): Platyhelminth Parasites of Amphibia. British Museum of Natural History. Oxford University Pres, London.

SAĞLAM, N., ARIKAN, H. (2006): Endohelminth parasites of the Marsh Frog Rana ridibunda from Hazar Lake, Turkey. Dis. Aquat. Org., 73: 253 - 260

SANChIS, V., Roig, J. M., CARretero, M. A., RocA, V., LlORENTE, G. A. (2000): Host-parasite relationships of Zootoca vivipara (Sauria: Lacertidae), in Pyreenes, North Spain. Folia Parasitol., 47: 118 - 122

Schad, G. A., Kuntz, R. E., Wells, W. H. (1960): Nematode parasites from Turkish vertebrates: an annotated list. Can. J. Zoolog., 38: 949 - 963

SEY, O., EORY, K. (1992): Helminth parasites of amphibians of the Lake Balaton area. Misc. Zool. Hung. 7: $5-8$ Shimalov, V. V., Shimalov, V. T. (2000): Helminth fauna of snakes (Reptilia, Serpentes) in Belorussian Pole- 
sye. Parasitol. Res., 86: 340 - 341

Shimalov, V. T. Shimalov, V. V., Shimalov, A. V. (2000): Helminth fauna of lizards in the southern Part of Belarus. Parasitol. Res., 86: 343. DOI: 10.1007/s004360 050057

Shimalov, V. V., Shimalov, V. T. (2001): Helminth fauna of toads in Belorussian Polesie. Parasitol. Res., 87: 84. DOI: $10.1007 / \mathrm{s} 004360000266$

Shimalov, V. V., Shimalov, V. T. Shimalov, A. V. (2001): Helminth fauna of newts in Belarusian Polesie. Parasitol. Res., 87: 356. DOI: 10.1007/PL00008592

Van Sluys, M., Schittini, G., Marra, R. V., AzeVedo, A., Vicente, J. J., VRCiBradic, D. (2006): Body size, diet and endoparasites of the microhylid frog Chiasmocleis capixaba in an Atlantic Forest Area of Southern Bahiastate, Brazil. Brazil. J. Biol., 66 (1A): $167-173$

VAshetKo, E. V., Siddikov, B. H. (1999): The effect of the ecology of toads on the distribution of helminths. Turk. J. Zool., 23: $107-110$

Veith, M., ERPelding, G. (1995): Presence of Pomphorhynchus laevis in Salamandra salamandra. J. Helminthol., 69: $267-268$

VojtKovÁ, L., VoJTeK, J. (1975): Die Trematoden der Amphibian inder Tschechowakei (Motolice Obojzivelniku CSSR) II Larval stadian (Mesocercariana und metacercarien) Folia Biol-Praque 15: 1 - 86

WALtON, A. C. (1933): The nematoda as parasites of Amphibia. J. Parasitol., 20: (1) 1 - 33

Yamaguti, S. (1958): Systema Helminthum. The digenea trematodes of vertebrates, Vol I. Part II, Digenea of Amphibians. Intersciences Publishers Ltd., London, England Yamaguti, S. (1961): Systema Helminthum. The nematodes of vertebrates. Vol III., Part II. Nematodes of Amphibians. Intersciences Publishers Ltd. London, England YAmaguti, S. (1963): Systema Helminthum. Acanthocephala. Vol.V. Intersciences Publishers, London, England Yildirimhan, H. S., UĞURtaş, İ. H., Altunel, F. N. (1996): An investigation on helminths of Rana ridibunda Pallas, 1771 (marsh frog). Acta Parasitol. Tur., 20: 113 130 (in Turkish, with English abstract)

Yildirimhan, H. S., UĞURTAŞ, İ. H. Altunel, F. N. (1997a): An investigation on parasitic helminths of Rana macrocnemis Boulenger, 1885 (Uludag frog). Turk. J. Zool., 21: $467-473$

YILDIRIMHAN, H. S., OĞUZ, M. C., UĞURTAŞ, İ. H. (1997b): An investigation on the nematodes of some tailless frogs (Rana ridibunda, Bufo bufo and Pelobates syriacus) collected from the Bursa regions. Hacettepe Fen ve Müh. Bil. Derg., 18: 45 - 58 (in Turkish with English abstract) YILDIRIMHAN, H. S. (1999a): Researches on parasitic helminths of Bufo viridis Laurenti, 1768 (Anura: Amphibia). Turk. J. Zool, 23: 177 - 195
YILDIRIMHAN, H. S. (1999b): Helminth fauna of lizards species belonging to Lacertidae (Reptilia) family distributed in Bursa and its around (PhD thesis). Uludag University, Institute of Science. 120 pp, Bursa (in Turkish, with English abstract)

Yildirimhan, H. S., AYdoĞdU, A., UĞURTAŞ, I. H., Altunel, F. N. (2001a): Helminth fauna of Bombina bombina (Linnaeus, 1761) (Fire-Bellied Toad) collected from Sakarya and Edirne (Turkey). Acta Parasitol. Tur., 25: 308 - 311 (in Turkish, with English abstract)

Yildirimhan, H. S., AYdOĞDU, A., UĞURTAŞ, I. H., Altunel, F. N. (2001b): An investigation on Plathelminth and Acanthocephala of Mertensiella caucasica (Caucasian Salamander).Acta Parasitol. Tur., 25: 393 - 397 (in Turkish, with English abstract)

YILDIRIMHAN, H. S., KARADENIZ, E., GÜRKAN, E., KOYUN, M. (2005a): Metazoon parasites of the Marsh frog (Rana ridibunda Pallas, 1771; Anura) collected from the different regions in Turkey. Acta Parasitol. Tur., 29 (2): 135 - 139 (in Turkish, with English abstract)

Yildirimhan, H. S., Bursey C. R., GoldberG, S. R. (2005b): Helminth parasites of the Caucasian salamander, Mertensiella caucasica, from Turkey. Comp. Parasitol., 72 (1): 75 - 87. DOI: 10.1654/4152

Yildirimhan, H. S., GoldberG, S. R. Bursey, C. R. (2006a): Helminth parasites of the Banded Frog, Rana camerani (Ranidae) from Turkey. Comp. Parasitol., 73 (2): 222 - 236. DOI: 10.1654/4229.1

Yildirimhan, H. S., Bursey, C. R. GoldberG S. R. (2006b): Helminth parasites of the Taurus frog, Rana holtzi, and the Uludag frog, Rana macrocnemis, with remarks on the helminth community of Turkish anurans. Comp. Parasitol., 73 (2): 237 - 248. DOI: 10.1654/4191.1 Yildirimhan, H. S., Altunel, F. C., UĞurtaș. İ. H. (2006 c): Helminth parasites of Hyla arborea (Linneaus, 1758) (Tree Frog) collected from Bursa, Edirne and Sakarya. Acta Parasitol. Tur, 30 (1): 56 - 59 (In Turkish, with English abstract)

Yildirimhan, H. S., KARADENIZ, E. (2007): Helminth Parasites of the Common Toad, Bufo bufo (Linnaeus, 1758) (Anura: Bufonidae) from Northeast Turkey. Comp. Parasitol., 74 (1): 176 - 178. DOI: 10.1654/4246.1

YILDIZ, K. (2003): Helminth infections in tench (Tinca tinca) from Kapulukaya Dam Lake. Turk. J. Vet. Anim. Sci., 27: 671 - 675 (In Turkish, with English abstract)

YILDIZ, K., ÇAVUŞOĞLU, K. (2003): A Scanning electron microscope examination of Pomphorhynchus laevis. Turk. J. Vet. Anim. Sci., 27: 1357 - 1360 (In Turkish, with English abstract)

ZiolkowskA, M., Rokicki, J. (2003): An Attempt to Determine the Intermediate Host for Pomphorhynchus laevis (Acanthocephala) in the Baltic Sea. Acta Ichtyol. Pisc., 37 (3): $159-161$

ACCEPTED MARCH 24, 2010 